Open Access

\title{
Seroprevalence of Toxoplasma gondii infection among patients with hand, foot and mouth disease in Henan, China: a hospital-based study
}

Shuai Wang ${ }^{1 \dagger}$, Chunwei Lan ${ }^{1,2+}$, Luwen Zhang ${ }^{1}$, Haizhu Zhang ${ }^{1}$, Zhijun Yao ${ }^{1}$, Dong Wang ${ }^{1}$, Jingbo Ma ${ }^{1}$, Jiarong Deng ${ }^{1}$ and Shiguo Liu $^{1 *}$

\begin{abstract}
Background: The prevalence of infection with Toxoplasma gondii (T. gondii) in humans has been increasing in China due to the growing number of cats in the country. Hand, foot and mouth disease (HFMD) is a serious public health issue in China and still one of the leading causes of child mortality. However, little is known about the epidemiology of $T$. gondii infection among HFMD patients.

Methods: A case-control study of 281 HFMD patients from the First People's Hospital of Pingdingshan in Pingdingshan city, Henan province, central China, and 222 controls from Pingdingshan city was conducted. Anti-T. gondii antibodies were serologically detected using the enzyme-linked immunosorbent assay.

Results: We found that the overall anti-T. gondii immunoglobulin $\mathrm{G}(\mathrm{IgG})$ antibody prevalence among HFMD patients was $12.46 \%$, which was significantly higher than that in clinically healthy children (1.80\%). The highest T. gondii seroprevalence was detected in critical cases (22.58 \%), followed by severe cases (11.50\%), and the lowest was detected in mild cases (8.33\%).

Conclusion: The present study is the first survey of T. gondii seroprevalence among HFMD patients in China; $12.46 \%$ were defined as seropositive. It is imperative that improved integrated measures are taken to prevent and control T. gondii infection among HFMD patients.
\end{abstract}

Keywords: Toxoplasma gondii, Hand, Foot and mouth disease, Seroprevalence, Enzyme-linked immunosorbent assay, China

\section{Multilingual abstracts}

Please see Additional file 1 for translations of the abstract into the six official working languages of the United Nations.

\section{Background}

Hand, foot and mouth disease (HFMD) is a common viral illness mainly caused by coxsackievirus A16 (Cox A16) and enterovirus 71 (EV71) [1, 2] infections.

\footnotetext{
*Correspondence: Isg1963@163.com

${ }^{\dagger}$ Equal contributors

'Department of Human Parasitology, Xinxiang Medical University, Xinxiang, Henan 453003, PR China

Full list of author information is available at the end of the article
}

Children under five years of age are more susceptible to HFMD, as over $50 \%$ of children in this age bracket lack the neutralizing antibodies that protect against EV71 and Cox A16 infections [1, 3, 4].

Symptoms of HFMD include fever, headache and poor appetite, followed by an intensely sore throat and a rash with very small blisters on the hands, feet and diaper area. A small number of children suffer complications such as myocarditis, pulmonary edema and aseptic meningoencephalitis, which can be fatal [5-7]. Occasionally, adults can contract HFMD $[1,8]$.

Since the 1980s, a number of severe HFMD outbreaks have been documented and HFMD remains a significant public health challenge, especially in the Asia-Pacific region

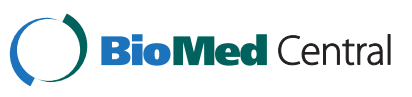

(c) 2015 Wang et al. Open Access This article is distributed under the terms of the Creative Commons Attribution 4.0 International License (http://creativecommons.org/licenses/by/4.0/), which permits unrestricted use, distribution, and reproduction in any medium, provided you give appropriate credit to the original author(s) and the source, provide a link to the Creative Commons license, and indicate if changes were made. The Creative Commons Public Domain Dedication waiver (http://creativecommons.org/publicdomain/zero/1.0/) applies to the data made available in this article, unless otherwise stated. 
[9-13]. Since the initiation of the national surveillance for HFMD in China in 2008, the incidence of reported cases has sharply increased, with more than seven million cases reported, including approximately 2,500 deaths [11]. From 1 January 2008 to 31 December 2013, a total of 400,264 HFMD cases have been reported in the Henan province alone, including 22,309 severe and 141 fatal cases [11].

According to the guidelines of the National Health and Family Planning Commission (NHFPC) of the People's Republic of China for diagnosis and treatment of HFMD (2010 edition, http://www.nhfpc.gov.cn/), HFMD cases are classified as mild, severe or critical. In mild cases, a skin rash appears on the hands, feet, mouth or buttocks of the patient, with or without fever. In severe cases, in addition to the above, patients can experience deflated moods or lethargy; become easily frightened or delirious; develop headaches; suffer from vomiting, limb shaking, myoclonus, nystagmus, ataxia, eye movement disorders, weakness or acute flaccid paralysis; and/or experience convulsions. There are also visible signs of meningeal irritation, and tendon reflexes are diminished or absent. In critical cases, at least one of the following has to occur: frequent convulsions, coma and brain herniation; dyspnea, cyanosis, bloody foam sputum, pulmonary rales, etc.; or shock and other signs of circulatory insufficiency.

Toxoplasma gondii ( $T$. gondii) is a protozoan parasite that can infect virtually all warm-blooded animals, including humans. It has been estimated that one-third of the world's population has been infected with this parasite $[14,15]$. The prevalence of infection with $T$. gondii in humans has been increasing in China due to the growing number of cats in the country [16]. Although in immunocompetent individuals, most $T$. gondii infections are asymptomatic, the infection can lead to serious diseases among immunocompromised patients such as HIV-positive and cancer patients, and transplant recipients [17-19].

Hand, foot and mouth disease is a serious public health issue in China and still one of the leading causes of child mortality. Numerous studies have shown that Th1/Th2 and Th17/Treg imbalances exist in HFMD patients [20]. Children with HFMD caused by the EV71 infection can suffer from functional disorders of cell, humoral and innate immunity [21]. Any of these functional disorders can increase the chance of infection with opportunistic pathogens such as T. gondii [22]. Yet, epidemiological knowledge about the prevalence of $T$. gondii infection among HFMD patients is unavailable in China. The present study is the first to estimate the prevalence of $T$. gondii infection among HFMD patients in China.

\section{Methods}

Sample collection

A total of 281 clinically diagnosed HFMD patients (24 mild cases, 226 severe cases and 31 critical cases) from the First People's Hospital of Pingdingshan in Pingdingshan city, Henan province, central China, were enrolled in the study from March to April 2014. The HFMD patients were classified into mild, severe or critical cases, according to the guidelines of the NHFPC of the People's Republic of China for diagnosis and treatment of HFMD (2010 edition, http://www.nhfpc.gov.cn/). Demographic data such as gender and age were obtained. Two hundred and twenty-two control subjects, matched with HFMD patients by age, gender and residence, were also included in the study. They were clinically healthy children without EV71, CoxA16 or other enterovirus infections, all from Pingdingshan city.

Samples of approximately $4 \mathrm{ml}$ of venous blood were taken from each patient and the control subjects with informed consent. All blood samples were labelled individually and cooled using ice packs to maintain the temperature at $4{ }^{\circ} \mathrm{C}$ during transportation to the laboratory.

\section{Serum sample preparation}

Blood samples were centrifuged and sera were recovered and transferred to $1.5 \mathrm{ml}$ Eppendorf Tubes ${ }^{\mathrm{Tx}}$. The serum samples were stored at $-80{ }^{\circ} \mathrm{C}$ until tested for $T$. gondii antibodies.

\section{Toxoplasma-lgG assessment}

Immunoglobulin G (IgG) antibodies of $T$. gondii were detected using a commercially manufactured enzymelinked immunosorbent assay (ELISA) kit (Zhuhai S.E.Z. Haitai Biological Pharmaceuticals Co., Ltd, Zhuhai, China). The manufacturer's instructions were followed to conduct the procedure. In brief, test serum (1:100 dilution) was added to each well in the coated plate and incubated for 30 minutes at $37^{\circ} \mathrm{C}$. After additional washing with a washing solution (PBS containing $0.05 \%$ Tween20, PBST), $50 \mu \mathrm{L}$ of peroxidase-conjugated antihuman IgG was added to the wells and incubated at $37^{\circ} \mathrm{C}$ for 30 minutes. This was followed by three washes using the washing solution. The colour reactions were developed by adding $50 \mu \mathrm{L}$ "A" solution $\left(\mathrm{H}_{2} \mathrm{O}_{2}\right)$ and $50 \mu \mathrm{L}$ "B" solution $\left(3,3^{\prime}, 5,5^{\prime}\right.$-Tetramethylbenzidine) at $37{ }^{\circ} \mathrm{C}$ for 10 minutes, and then the reaction was stopped by adding $50 \mu \mathrm{L}$ of stopping solution $\left(0.5 \mathrm{ml} / \mathrm{L} \mathrm{H}_{2} \mathrm{SO}_{4}\right)$. Microplates were read at an optical density (OD) of $450_{\mathrm{nm}}$ in the Model 550 microplate ELISA reader (Bio-Rad, Hercules, CA, USA), and ratios (OD450 $\mathrm{nm}$ value of serum sample/ OD450 $\mathrm{nm}$ value of negative control) were calculated after correcting for the OD450 $\mathrm{nm}$ value of the blank control (without serum and peroxidase-conjugated anti-human IgG). Test serum samples were considered positive when the ratio was $\geq 2.1$. 


\section{Statistical analysis}

Statistical analysis was performed using the SPSS version 20 software for Windows (SPSS Inc., Chicago, Illinois, USA). The chi-square test was used to perform statistical analyses of $T$. gondii prevalence using different variables. Differences were considered statistically significant if $p<0.05$.

\section{Ethical statement}

The study was reviewed and approved by the Ethical Review Committee of the Xinxiang Medical University (reference no. 2013008). Written informed consent was obtained from all participants.

\section{Results}

As shown in Table 1, anti-T. gondii IgG antibodies were detectable in the sera of 35 HFMD cases (35/281), with an overall seroprevalence of $12.46 \%$. For clinically healthy children, a total of four (1.80\%) of the 222 samples were positive for anti-T. gondii IgG antibodies. The seropositive rate of the former was significantly higher than that of the latter $\left(X^{2}=19.681, p<0.001\right)$.

In HFMD cases, the seroprevalence of $T$. gondii in males was $12.30 \%(23 / 187)$ and $12.77 \%(12 / 94)$ in females. No significant difference between genders was observed $\left(X^{2}=0.012, p=0.911\right)$. The highest seroprevalence of $T$. gondii infection was found among children in the age group of one to two years $(16.09 \%, 14 / 87)$ and the lowest was found among children in the age group of < one year $(8.87 \%, 11 / 124)$. However, the difference between age groups was not significantly significant $(p>0.05)$ (see Table 1$)$.

Table 2 shows the clinical diagnosis data of HFMD patients based on the severity of their disease. The seroprevalence of $T$. gondii infection was highest in critical cases $(22.58 \%, 7 / 31)$, followed by severe cases (11.50\%, 26/226), and was the lowest in mild cases
(8.33 \%, 2/24). However, no statistically significant differences were observed among the three groups $\left(X^{2}=3.476, p=0.176\right)$.

\section{Discussion}

Infection with $T$. gondii in humans is common all around the world, with prevalence rates varying depending on the environment, people's eating habits and age [23]. The two nation-wide surveys carried out in 1995 and 2004 reported that the prevalence of $T$. gondii infection in the Chinese population was around $7 \%$ [24]. In 2010, the infection rate of T. gondii in the Chinese population increased to $12.3 \%$ [25].

In the present study, $12.46 \%(35 / 281)$ of HFMD patients were seropositive for $T$. gondii. This is higher than that reported in HIV-positive patients (3.5-7.7\%) $[26,27]$, similar to the general infection rate of $T$. gondii (12.3\%) [25], and lower than that observed in female sterility patients $(15.9 \%)$ [28], patients in intensive care units (18.78 \%) [23], psychiatric patients (17.30\%) [29], cancer patients $(35.56 \%)$ and dialysis patients (27.3\%) $[30,31]$ in China.

Additionally, the seropositive rate of $T$. gondii infection among HFMD patients was significantly higher than that of clinically healthy children $(1.80 \%)$. Children with HFMD caused by the EV71 infection can suffer from functional disorders of cell, humoral and innate immunity $[20,21]$, which can reduce the body's resistance to opportunistic pathogens such as $T$. gondii and predispose them to develop secondary toxoplasmosis. Latent T. gondii infections could also be activated. However, whether the prevalence of $T$. gondii infection can increase the risk of contracting HFMD and affect the disease outcome of HFMD patients requires further study.

In the present study, female HFMD patients had a higher prevalence than male patients, although the difference was not significant $(p>0.05)$. Gender was thus

Table 1 Seroprevalence of Toxoplasma gondii infection in the study populations in China

\begin{tabular}{|c|c|c|c|c|c|c|c|c|}
\hline \multirow[t]{2}{*}{ Variable } & \multicolumn{3}{|l|}{ HFMD patients } & \multicolumn{3}{|c|}{ Control subjects } & \multicolumn{2}{|c|}{ HFMD patients vs control subjects } \\
\hline & No. examined & No.of positive & Prevalence (\%) & No. examined & No.of positive & Prevalence (\%) & $x^{2}$ & $P$-value \\
\hline \multicolumn{9}{|l|}{ Gender } \\
\hline Male & 187 & 23 & 12.30 & 130 & 4 & 3.08 & 8.371 & 0.004 \\
\hline Female & 94 & 12 & 12.77 & 92 & 0 & 0 & 12.555 & $<0.001$ \\
\hline \multicolumn{9}{|l|}{ Age (year) } \\
\hline$<1$ & 124 & 11 & 8.87 & 64 & 0 & 0 & 4.527 & 0.033 \\
\hline $1 \sim 2$ & 87 & 14 & 16.09 & 56 & 0 & 0 & 9.989 & 0.002 \\
\hline $2 \sim 3$ & 44 & 6 & 13.64 & 52 & 0 & 0 & 5.415 & 0.02 \\
\hline$>3$ & 26 & 4 & 15.38 & 50 & 4 & 8.00 & 0.362 & 0.548 \\
\hline Total & 281 & 35 & 12.46 & 222 & 4 & 1.80 & 19.681 & $<0.001$ \\
\hline
\end{tabular}


Table 2 Clinical diagnosis and seroprevalence of T. gondii in HFMD patients in China

\begin{tabular}{lllc}
\hline Clinical & \multicolumn{3}{l}{ Patients with anti-T. gondii antibodies } \\
\cline { 2 - 4 } & No.examined & No.of positive & Prevalence (\%) \\
\hline Mild cases & 24 & 2 & $8.33^{\mathrm{a}}$ \\
Severe cases & 226 & 26 & $11.50^{\mathrm{a}}$ \\
Critical cases & 31 & 7 & $22.58^{\mathrm{a}}$ \\
Total & 281 & 35 & 12.46 \\
\hline
\end{tabular}

Values bearing a different superscript letter (a) within a column differ significantly from one another $(p<0.05)$

not significantly associated with the presence of anti- $T$. gondii antibodies $(P>0.05)$, which was consistent with other reports [32, 33].

Rates of $T$. gondii infection increased with age in this study, however, the difference between age groups was not significantly significant $(p>0.05)$. A hypothesis would be that the increase is a reflection of increasing 'exposure years' as the children get older [32]. The results provided further evidence for the increased risk of T. gondii infection with acquisition of age. Seroprevalence of $T$. gondii infection was highest in critical HFMD cases, followed by severe cases, and the lowest was detected in mild cases, with percentages of $\mathrm{CD} 4^{+}, \mathrm{CD}^{+} \mathrm{T}$ and $\mathrm{CD} 16^{+} 56^{+}$cells (natural killer cells) continuously decreasing as the disease worsens [21]. Additionally, $\mathrm{CD}^{+}$and $\mathrm{CD}^{+} \mathrm{T}$ cells and natural killer cells play important roles in the process of anti- $T$. gondii infection $[34,35]$. These findings might explain why the probability of seroprevalence with $T$. gondii infection increases with the severity of the HFMD disease. With respect to the increased numbers of HFMD patients worldwide, toxoplasmosis should be considered as potentially associated with HFMD in humans.

\section{Conclusion}

The present study revealed for the first time the seroprevalence of $T$. gondii infection among HFMD patients in the Henan province, China. Further studies should be conducted to estimate the prevalence of $T$. gondii infection among HFMD patients in other provinces of China, with increased sample sizes.

\section{Additional files}

Additional file 1: Multilingual abstracts in the six official working languages of the United Nations. (PDF $341 \mathrm{~kb}$ )

\section{Abbreviations}

Cox A16: coxsackievirus A16; ELISA: enzyme-linked immunosorbent assay; EV71: enterovirus 71; HFMD: hand, foot and mouth disease; IgG: immunoglobulin G; NHFPC: National Health and Family Planning Commission; OD: optical density; T. gondii: Toxoplasma gondii.
Competing interests

The authors declare that they have no competing interests.

\section{Authors' contributions}

SL conceived and designed the study. SW, CL, LZ, HZ and JD collected the samples and performed the experiments. SW, ZY, DW and JM performed the statistical analyses and drafted the paper. All authors read and approved the final paper.

\section{Acknowledgements}

This study was supported by the Key Scientific and Technological Project of Xinxiang city (no. ZG15014), the Science and Technology Research Key Project of Education Department of Henan province (no. 13A310845) and the Doctoral Scientific Research Activation Foundation of Xinxiang Medical University (no. XYBSKYZZ201504)

\section{Author details}

'Department of Human Parasitology, Xinxiang Medical University, Xinxiang, Henan 453003, PR China. 'First People's Hospital of Pingdingshan,

Pingdingshan, Henan 467000, PR China.

Received: 23 July 2015 Accepted: 10 October 2015

Published online: 10 December 2015

\section{References}

1. Wang $X$, Xing M, Zhang C, Yang Y, Chi Y, Tang X, et al. Neutralizing antibody responses to enterovirus and adenovirus in healthy adults in China. Emerg Microb Infect. 2014;3:e30.

2. Liu Y, Wang X, Pang C, Yuan Z, Li H, Xue F. Spatio-temporal analysis of the relationship between climate and hand, foot, and mouth disease in Shandong province, China, 2008-2012. BMC Infect Dis. 2015;15:146.

3. Xu M, Yu W, Tong S, Jia L, Liang F, Pan X. Non-Linear Association between Exposure to Ambient Temperature and Children's Hand-Foot-and-Mouth Disease in Beijing, China. PloS one. 2015;10:e0126171.

4. Di B, Zhang Y, Xie H, Li X, Chen C, Ding P, et al. Circulation of Coxsackievirus A6 in hand-foot-mouth disease in Guangzhou, 2010-2012. Virol J. 2014;11:157.

5. Irving S, Barclay-Buchanan C. Onychomadesis: A Rare Sequela of Hand, Foot, and Mouth Disease. Internet J Emerg Med. 2015.

6. Liu SL, Pan H, Liu P, Amer S, Chan TC, Zhan J, et al. Comparative epidemiology and virology of fatal and nonfatal cases of hand, foot and mouth disease in mainland China from 2008 to 2014. Rev Med Virol. 2015;25:115-28.

7. Kashyap RR, Kashyap RS. Hand, foot and mouth disease-a short case report. J Clin Exp Dent. 2015;7:e336-8.

8. Downing C, Ramirez-Fort MK, Doan HQ, Benoist F, Oberste MS, Khan F, et al. Coxsackievirus A6 associated hand, foot and mouth disease in adults: clinical presentation and review of the literature. J Clin Virol. 2014;60:381-6.

9. Sarma N. Hand, foot, and mouth disease: current scenario and Indian perspective. Indian J Dermatol Venereol Leprology. 2013;79:165-75.

10. Ling BP, Jalilian FA, Harmal NS, Yubbu P, Sekawi Z. Detection and characterization of viruses causing hand, foot and mouth disease from children in Seri Kembangan, Malaysia. Trop Biomed. 2014;31:654-62.

11. Huang X, Wei H, Wu S, Du Y, Liu L, Su J, et al. Epidemiological and etiological characteristics of hand, foot, and mouth disease in Henan, China, 2008-2013. Sci Rep. 2015;5:8904

12. Wu Y, Yeo A, Phoon MC, Tan EL, Poh CL, Quak SH, et al. The largest outbreak of hand; foot and mouth disease in Singapore in 2008: the role of enterovirus 71 and coxsackievirus A strains. Int J Infect Dis. 2010;14:e1076-81.

13. Edmond M, Wong C, Chuang SK. Evaluation of sentinel surveillance system for monitoring hand, foot and mouth disease in Hong Kong. Publ Health. 2011;125:777-83.

14. Wang S, Zhao GW, Wang W, Zhang ZC, Shen B, Hassan IA, et al. Pathogenicity of Five Strains of Toxoplasma gondii from Different Animals to Chickens. Kor J Parasitol. 2015;53:155-62.

15. Hassan IA, Wang $S$, Xu L, Yan R, Song X, Li X. DNA vaccination with a gene encoding Toxoplasma gondii Deoxyribose Phosphate Aldolase (TgDPA) induces partial protective immunity against lethal challenge in mice. Parasites Vectors. 2014;7:431.

16. Zhou P, Chen Z, Li HL, Zheng H, He S, Lin RQ, et al. Toxoplasma gondii infection in humans in China. Parasites Vectors. 2011;4:165. 
17. Hassan IA, Wang S, Xu L, Yan R, Song X, Li X. Immunoglobulin and cytokine changes induced following immunization with a DNA vaccine encoding Toxoplasma gondii selenium-dependent glutathione reductase protein. Exp Parasitol. 2014;146:1-10.

18. Kodym P, Maly M, Beran O, Jilich D, Rozsypal H, Machala L, et al. Incidence, immunological and clinical characteristics of reactivation of latent Toxoplasma gondii infection in HIV-infected patients. Epidemiol Infect. 2015;143:600-7.

19. Wang L, He LY, Meng DD, Chen ZW, Wen H, Fang GS, et al. Seroprevalence and genetic characterization of Toxoplasma gondii in cancer patients in Anhui Province, Eastern China. Parasites Vectors. 2015;8:162.

20. Li S, Cai C, Feng J, Li X, Wang Y, Yang J, et al. Peripheral T lymphocyte subset imbalances in children with enterovirus 71-induced hand, foot and mouth disease. Virus Res. 2014;180:84-91.

21. Ke JW, Chen Q, Duan R. Study of the changes of immune status in children with HFMD caused by EV71 virus. Exp Lab Med. 2014;32:5-7.

22. Zhang H, Hu X, Liu X, Zhang R, Fu Q, Xu X. The Treg/Th17 imbalance in Toxoplasma gondii-infected pregnant mice. Am J Reprod Immunol. 2012;67:112-21.

23. Zhang $Y B$, Cong $W, L i Z T, B i X G$, Xian $Y$, Wang $Y H$, et al. Seroprevalence of Toxoplasma gondii Infection in Patients of Intensive Care Unit in China: A Hospital Based Study. BioMed Res Int. 2015;2015:908217.

24. Coordinating Office of the National Survey on the Important Human Parasitic D. A national survey on current status of the important parasitic diseases in human population. Chinese J Parasitol Parasitic Dis. 2005;23:332-40.

25. Xiao Y, Yin J, Jiang N, Xiang M, Hao L, Lu H, et al. Seroepidemiology of human Toxoplasma gondii infection in China. BMC Infect Dis. 2010;10:4.

26. Zhang $X, X u$ J, Peng $H, M a Y$, Han L, Ruan $Y$, et al. HCV coinfection associated with slower disease progression in HIV-infected former plasma donors naive to ART. PLoS One. 2008;3:e3992.

27. Xiao J, Gao G, Li Y, Zhang W, Tian Y, Huang Y, et al. Spectrums of opportunistic infections and malignancies in HIV-infected patients in tertiary care hospital, China. PloS one. 2013;8:e75915.

28. Li S, Cui L, Zhao J, Dai P, Zong S, Zuo W, et al. Seroprevalence of Toxoplasma gondii infection in female sterility patients in China. J Parasitol. 2011;97:529-30.

29. Cong W, Dong W, Bai L, Wang XY, Ni XT, Qian AD, et al. Seroprevalence and associated risk factors of Toxoplasma gondii infection in psychiatric patients: a case-control study in eastern China. Epidemiol Infect. 2015;1:7.

30. Tong DS, Yang J, Xu GX, Shen GQ. Serological investigation on Toxoplasma gondii infection in dialysis patients with renal insufficiency. Chinese J Schistosomiasis Control. 2011;23:144-53.

31. Cong W, Liu GH, Meng QF, Dong W, Qin SY, Zhang FK, et al. Toxoplasma gondii infection in cancer patients: prevalence, risk factors, genotypes and association with clinical diagnosis. Cancer Lett. 2015;359:307-13.

32. Meng QF, You HL, Zhou N, Dong W, Wang WL, Wang WL, et al. Seroprevalence of Toxoplasma gondii antibodies and associated risk factors among children in Shandong and Jilin provinces, China. Int J Infect Dis. 2015;30:33-5.

33. Kamani J, Mani AU, Egwu GO, Kumshe HA. Seroprevalence of human infection with Toxoplasma gondii and the associated risk factors, in Maiduguri, Borno state, Nigeria. Ann Trop Med Parasitol. 2009;103:317-21.

34. Dupont CD, Christian DA, Hunter CA. Immune response and immunopathology during toxoplasmosis. Semin Immunopathol. 2012;34:793-813.

35. Dupont CD, Christian DA, Selleck EM, Pepper M, Leney-Greene M, Harms Pritchard G, et al. Parasite fate and involvement of infected cells in the induction of CD4+ and CD8+ T cell responses to Toxoplasma gondii. PLoS Pathog. 2014;10:e1004047.

\section{Submit your next manuscript to BioMed Central and take full advantage of:}

- Convenient online submission

- Thorough peer review

- No space constraints or color figure charges

- Immediate publication on acceptance

- Inclusion in PubMed, CAS, Scopus and Google Scholar

- Research which is freely available for redistribution 